\title{
Internalization of paramagnetic phosphatidylserine-containing liposomes by macrophages
}

\author{
Tessa Geelen, Sin Yuin Yeo, Leonie EM Paulis, Lucas WE Starmans, Klaas Nicolay and Gustav J Strijkers*
}

\begin{abstract}
Background: Inflammation plays an important role in many pathologies, including cardiovascular diseases, neurological conditions and oncology, and is considered an important predictor for disease progression and outcome. In vivo imaging of inflammatory cells will improve diagnosis and provide a read-out for therapy efficacy. Paramagnetic phosphatidylserine (PS)-containing liposomes were developed for magnetic resonance imaging (MRI) and confocal microscopy imaging of macrophages. These nanoparticles also provide a platform to combine imaging with targeted drug delivery.

Results: Incorporation of PS into liposomes did not affect liposomal size and morphology up to $12 \mathrm{~mol} \%$ of PS. Liposomes containing 6 mol\% of PS showed the highest uptake by murine macrophages, while only minor uptake was observed in endothelial cells. Uptake of liposomes containing 6 mol\% of PS was dependent on the presence of $\mathrm{Ca}^{2+}$ and $\mathrm{Mg}^{2+}$. Furthermore, these 6 mol\% PS-containing liposomes were mainly internalized into macrophages, whereas liposomes without PS only bound to the macrophage cell membrane.

Conclusions: Paramagnetic liposomes containing 6 mol\% of PS for MR imaging of macrophages have been developed. In vitro these liposomes showed specific internalization by macrophages. Therefore, these liposomes might be suitable for in vivo visualization of macrophage content and for (visualization of) targeted drug delivery to inflammatory cells.
\end{abstract}

Keywords: Inflammation, Liposomes, MRI, Phosphatidylserine, Cell internalization

\section{Background}

Inflammation plays a crucial role in many pathologies, including cardiovascular diseases, neurological disorders and oncology, and is generally considered as an important predictor for disease progression and outcome [1,2]. Therefore, modulation of the inflammatory response by dedicated therapy is of particular interest.

The efficacy of traditional therapeutic compounds of low molecular weight is often limited by short blood circulation half-lives and adverse side effects due to non-specific systemic distribution and accumulation. Additionally, it is difficult to obtain quantitative information on the amount of drug accumulating in the diseased tissue. Drug delivery via a nanocarrier system provides an attractive alternative to

\footnotetext{
*Correspondence: g.j.strijkers@tue.nl

Department of Biomedical Engineering, Biomedical NMR, Eindhoven

University of Technology, PO Box 513, Eindhoven, MB 5600, the Netherlands
}

alleviate these drawbacks. For example, Doxil is a clinically approved nanocarrier system for cancer treatment, which consists of doxorubicin encapsulated in liposomes [3,4]. This formulation limits cardiotoxicity and prolongs the blood circulation half-life compared to free doxorubicin, which results in an enhanced time window for drug delivery and extravasation of the liposomes through the leaky tumor vasculature.

The surface composition of nanocarriers containing drugs can be tailored to tune clearance kinetics, for instance polyethylene glycol (PEG) is often incorporated to prolong the blood half-life [5]. Furthermore, the larger size of nanocarriers promotes a higher level of uptake in diseased tissues by the enhanced permeability and retention (EPR) effect [6,7]. Importantly, to address the inflammatory response in cardiovascular disease, the drug-containing nanocarriers should be delivered with high specificity to inflammatory cells in the diseased 
tissue. This can be achieved by introducing ligands that mediate nanocarrier recognition and internalization by the inflammatory cells.

An attractive route to target macrophages is by incorporation of the lipid phosphatidylserine (PS) in lipid-based nanoparticles, such as liposomes. In mammalian cells, PS is predominantly present in the inner leaflet of cell membranes. When a cell becomes apoptotic, PS is exposed on the outer leaflet of the cell membrane, which serves as a trigger for phagocytosis by macrophages $[8,9]$. The incorporation of PS in the liposomal membrane can therefore promote uptake by macrophages. Previously it was shown that incorporation of PS in liposomes indeed resulted in enhanced uptake by macrophages [10,11].

Also, magnetic resonance imaging (MRI) contrast agents can be incorporated to image drug delivery and obtain quantitative information on the local concentration of drugs at the target site [12,13]. Previously, Harel-Ader et al. developed liposomes with PS containing iron-oxides for MRI visualization of inflammatory cells in myocardial infarction [14] and Maiseyeu et al. described liposomes with PS containing Gd-DTPA-distearylamide for MR imaging of macrophages in atherosclerotic plaques [10]. However, a detailed characterization and optimization of MRI-detectable PS-containing liposomes, including the conditions under which they most effectively target macrophages and induce strongest contrast in MRI, is still lacking.

In this study, we therefore describe the design and characterization of MRI-detectable liposomes that are targeted to macrophages using PS. Liposomes containing different molar percentages of PS were prepared and liposome size and morphology were studied by dynamic light scattering (DLS) and cryogenic transmission electron microscopy (cryoTEM). Fluorescent labels, incorporated in the liposomes, enabled detailed analysis of liposome binding and internalization by macrophages using confocal laser scanning microscopy (CLSM) and fluorescence activated cell sorting (FACS). The ability of the liposomes to induce contrast changes in MR images was studied in macrophages and quantified on the basis of the measured changes in $T_{1}$ and $T_{2}$ relaxation times.

\section{Results}

\section{Characterization of liposomes}

Liposomes containing $0,6,12$ and $37 \mathrm{~mol} \%$ of phosphatidylserine (1,2-distearoyl-sn-glycero-3-phospho-L-serine = DSPS or PS) were prepared. In this paper we will refer to these liposomes as PC-L, PS-6-L, PS-12-L, and PS-37-L, respectively (see also Table 1 and Methods). Thin layer chromatography (TLC) confirmed the presence of PS in the liposome formulations by the appearance of a spot corresponding to DSPS (Figure 1a). The spot became
Table 1 Mol\% of lipids present in different liposome formulations

\begin{tabular}{lcccc}
\hline & PC-L & PS-6-L & PS-12-L & PS-37-L \\
\hline DSPS & 0 & 6 & 12 & 37 \\
DSPC & 37 & 31 & 25 & 0 \\
Gd-DOTA-DSPE & 25 & 25 & 25 & 25 \\
Cholesterol & 33 & 33 & 33 & 33 \\
PEG2000-DSPE & 5 & 5 & 5 & 5 \\
\hline
\end{tabular}

more intense with increasing mol\% PS in the lipid preparation mixture, which shows that PS was successfully incorporated in increasing amounts in the final liposome preparations up to $37 \mathrm{~mol} \%$.

Representative DLS spectra for the different types of liposomes are presented in Figure 1b. For all formulations a single dominant peak was observed, indicating a relatively narrow range of liposome diameters. PS-6-L and PS-12-L had the same mean hydrodynamic diameter as PC-L (Table 2). However, PS-37-L had a somewhat smaller diameter ( $\mathrm{p}<0.05$ vs. PC-L). Incorporation of PS resulted in a significant increase of the polydispersity index (PDI, p <0.05 vs. PC-L), which was also observed as a modest broadening of the DLS peaks (Figure 1b). We think that changes in the membrane rigidity or stability due to incorporation of PS leads to a smaller size after extrusion. Liposome morphology was investigated in more detail using cryoTEM (Figure 1c). CryoTEM images revealed predominantly single unilamellar liposomes for all formulations. For PC-L, PS-6-L and PS-12-L liposomes were spherical, whereas for PS-37-L occasionally non-spherical, deformed liposomes were observed (Figure 1c, black arrows).

The ability of the liposomes to generate contrast in MRI is determined by their potency to change the longitudinal $\left(\mathrm{T}_{1}\right)$ and transversal $\left(\mathrm{T}_{2}\right)$ relaxation times, which is expressed by the longitudinal $\left(r_{1}\right)$ and transversal $\left(r_{2}\right)$ relaxivity. The $r_{1}$ and $r_{2}$ of the liposomes at 9.4 $T$ and room temperature, normalized to $\mathrm{Gd}$ concentration, were $3.0-4.0 \mathrm{mM}^{-1} \cdot \mathrm{s}^{-1}$ and $42-60 \mathrm{mM}^{-1} \cdot \mathrm{s}^{-1}$, respectively (Table 2). Incorporation of PS did not significantly affect the longitudinal and transversal relaxivity. All liposome formulations displayed a similar relatively high $r_{2} / r_{1}$ ratio.

\section{Association of PS-containing liposomes with macrophages}

In vitro experiments were performed to determine which formulation of PS-containing liposomes resulted in highest association with mouse macrophages (RAW cells). RAW cells were incubated with PC-L, PS-6-L, PS-12-L and PS-37-L and association of liposomes with the macrophages was characterized by several readouts exploiting the various components of the liposomes, 


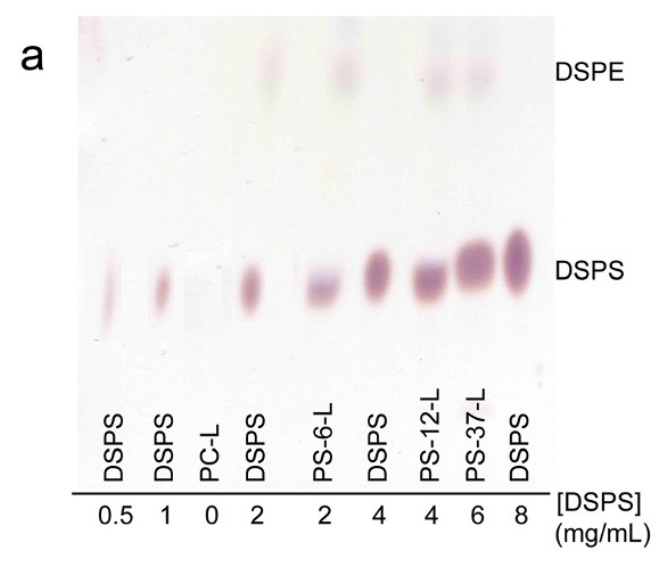

\section{b}
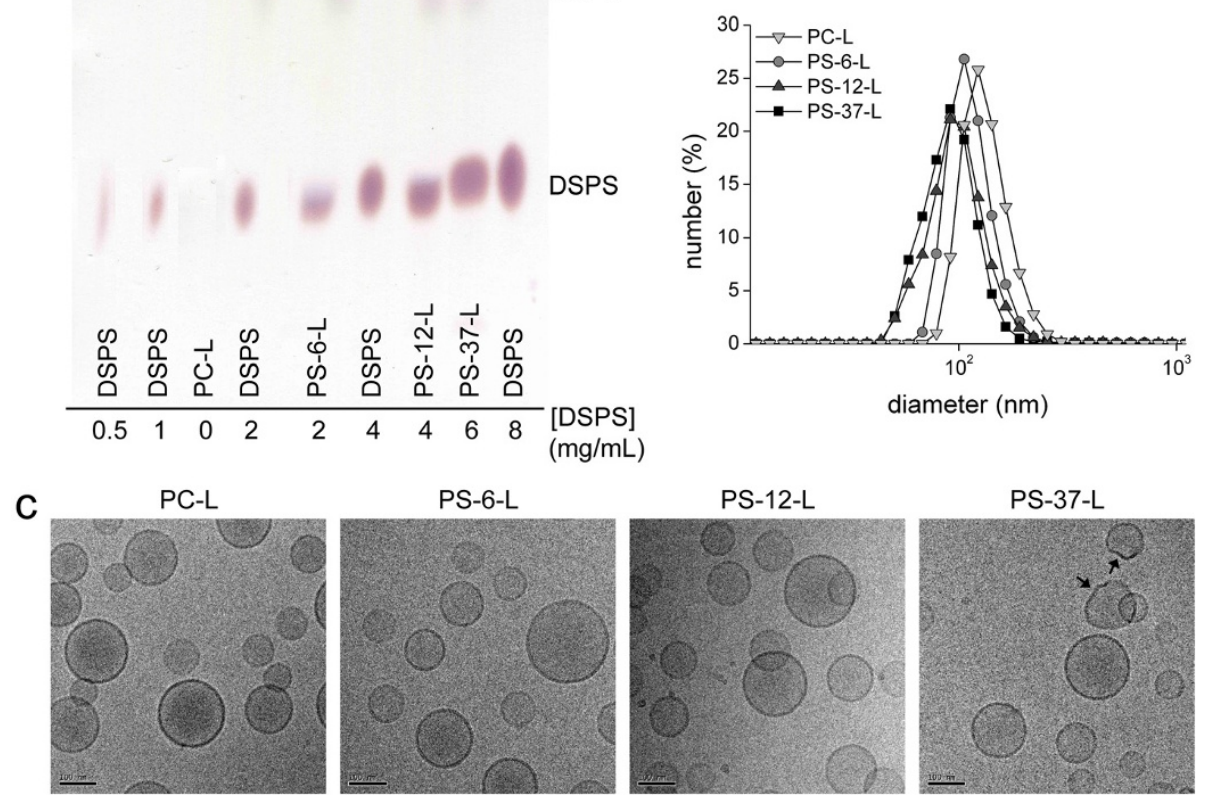

Figure 1 Characterization of paramagnetic liposomes. a) TLC of the different types of liposomes. b) Representative number-weighted DLS size distributions for all liposome formulations. c) cryoTEM images of the liposomes. The arrows point at non-spherical liposomes present in PS-37-L. Scale bar $=100 \mathrm{~nm}$.

including quantitative $\mathrm{T}_{1}$ and $\mathrm{T}_{2}$ mapping with MRI, quantitative $\mathrm{Gd}$ determinations by inductively coupled plasma mass spectrometry (ICP-MS), and CLSM.

Figure 2a shows results of quantitative Gd determinations of RAW cells, including untreated cells (no L) and cells incubated with PC-L, PS-6-L, PS-12-L or PS-37-L. Gd concentrations for the incubations with PS-12-L and PS-37-L were at the baseline level of PC-L non-specific uptake and untreated cells. Solely, incubation with PS-6$\mathrm{L}$ resulted in a significantly higher $\mathrm{Gd}$ concentration $(0.64 \pm 0.23 \mathrm{mM}, \mathrm{p}<0.05$ vs. no $\mathrm{L})$.

MRI measurements were performed at 9.4 $\mathrm{T}$ on the same cell pellets as for ICP-MS. MRI consisted of quantitative $\mathrm{T}_{1}$ and $\mathrm{T}_{2}$ mapping. The cells incubated with PS-6$\mathrm{L}$ could clearly be distinguished from untreated cells and cells incubated with other types of liposomes (Figure 2b).
Average $R_{1}\left(=1 / T_{1}\right)$ and $R_{2}\left(=1 / T_{2}\right)$ values for the different groups are summarized in Figure $2 \mathrm{c}$ and $2 \mathrm{~d}$, respectively. Incubation with liposomes always resulted in enhanced $R_{1}$ and $R_{2}$ values ( $p<0.05$ vs. no $L$ ). In agreement with quantitative $\mathrm{Gd}$ determinations, however less pronounced, both $R_{1}$ and $R_{2}$ were highest for the incubations with PS-6-L $\left(0.942 \pm 0.004 \mathrm{~s}^{-1}\right.$ and $37.3 \pm 1.2 \mathrm{~s}^{-1}$, respectively, $\mathrm{p}<0.05$ vs. all).

The relaxivities $r_{1}$ and $r_{2}$ in the cellular environment were estimated from the quantitative $\mathrm{Gd}$ determinations in relation to changes in $R_{1}$ and $R_{2}$ (Table 3). For PC-L, $r_{1}$ and $r_{2}$ were $1.9 \pm 0.3 \mathrm{mM}^{-1} \cdot \mathrm{s}^{-1}$ and $32.5 \pm 3.6 \mathrm{mM}^{-1} \cdot \mathrm{s}^{-1}$, respectively. For PS-6-L, $r_{1}$ and $r_{2}$ were $0.8 \pm 0.4 \mathrm{mM}^{-1} \cdot \mathrm{s}^{-1}$ and $16.3 \pm 5.8 \mathrm{mM}^{-1} \cdot \mathrm{s}^{-1}$. As shown previously, PC-L and PS-6-L relaxivities in aqueous solution were similar (Table 2). The lower cellular relaxivities for PS-6-L

Table 2 Characterization of liposome formulations

\begin{tabular}{lcccc}
\hline & PC-L & PS-6-L & PS-12-L & PS-37-L \\
\hline Hydrodynamic diameter $(\mathbf{n m})^{\mathbf{a}}$ & $136 \pm 3$ & $120 \pm 7$ & $111 \pm 10$ & $97 \pm 4^{*}$ \\
PDI $(-)^{\mathbf{a}}$ & $0.13 \pm 0.03$ & $0.28 \pm 0.05^{*}$ & $0.30 \pm 0.02^{*}$ & $0.30 \pm 0.19^{*}$ \\
$\mathbf{r}_{\mathbf{1}}\left(\mathbf{m M}^{-1} \cdot \mathbf{s}^{-1}\right)^{\mathbf{b}}$ & $3.0 \pm 0.1$ & $3.0 \pm 0.3$ & $3.4 \pm 0.4$ & $4.0 \pm 0.1$ \\
$\mathbf{r}_{\mathbf{2}}\left(\mathbf{m M}^{-1} \cdot \mathbf{s}^{-1}\right)^{\mathbf{b}}$ & $42.3 \pm 5.5$ & $51.5 \pm 4.5$ & $60.7 \pm 5.1$ & $46.7 \pm 4.2$ \\
$\mathbf{r}_{\mathbf{2}} / \mathbf{r}_{\mathbf{1}}^{\mathbf{b}}$ & $14.4 \pm 2.0$ & $18.1 \pm 2.6$ & $18.2 \pm 0.8$ & $11.8 \pm 0.9$ \\
\hline
\end{tabular}

${ }^{a} n=5$ for PC-L and PS-6-L and $n=4$ for PS-12-L and PS-37-L; ${ }^{b} n=4$ for PC-L and PS-6-L, and $n=3$ for PS-12-L and PS-37-L; ${ }^{*} p<0.05$ vs. PC-L, ANOVA with Bonferroni correction. 
a

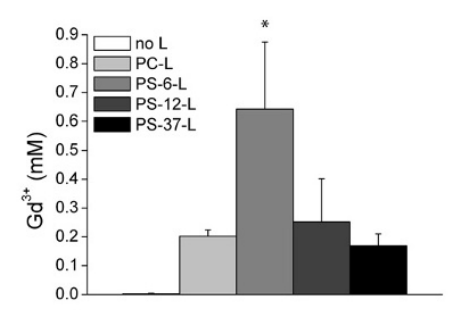

C

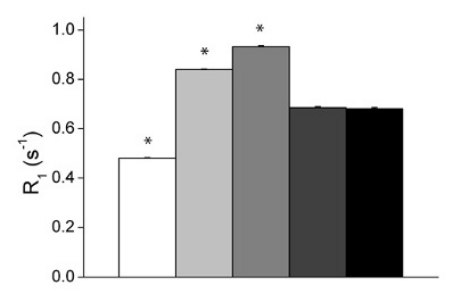

b

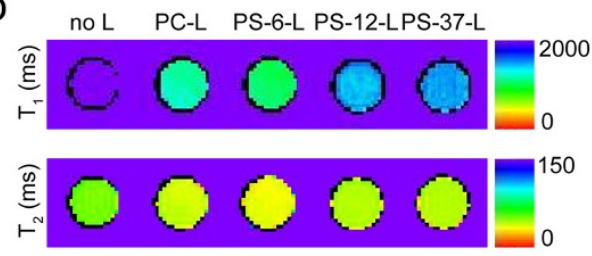

d

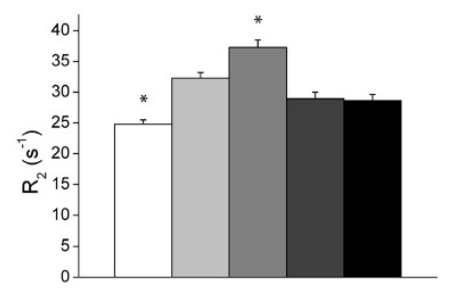

e
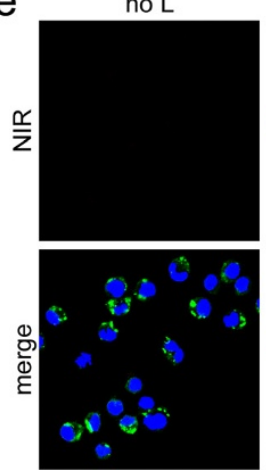

PC-L
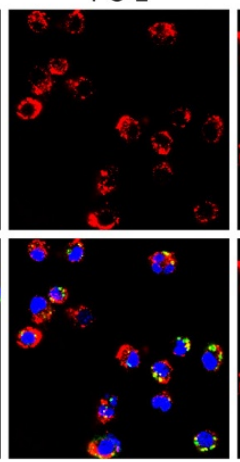

PS-6-L
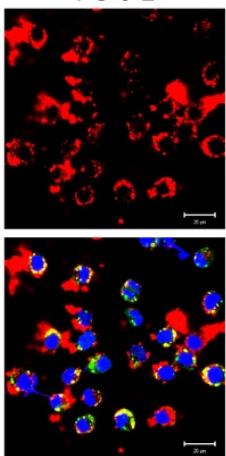

PS-12-L
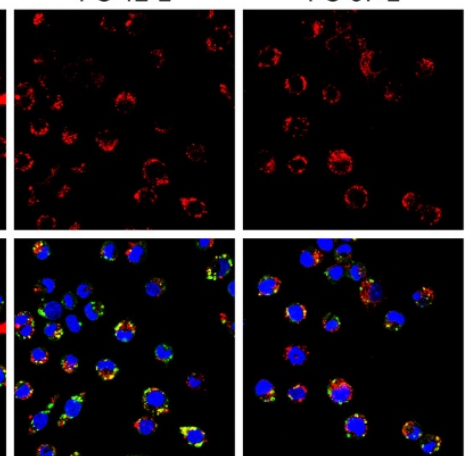

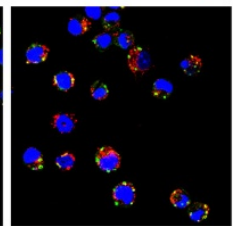

Figure 2 Association of PS-containing liposomes with RAW cells. a) Gd concentration of RAW cells incubated with different liposome formulations in RPMI medium as determined with ICP-MS ( $n=3$ /group, except for PS-6- $L n=2$ /group). * $p<0.05$ vs. no $L$, ANOVA with Bonferroni correction. b) Representative $T_{1}$ and $T_{2}$ maps of RAW cell pellets. c) Average $R_{1}$ and $\mathbf{d}$ ) average $R_{2}$ of cell pellets measured at $9.4 T$. ${ }^{*} p<0.05$ vs. all, ANOVA with Bonferroni correction. e) CLSM images of RAW cells. Top row: in red, the fluorescence signal of the NIR-lipids present in liposomes (laser intensity 2\% of maximal intensity). Bottom row: NIR signal (in red) merged with signal of labeled macrophage CD68 (in green) and cell nuclei (in blue). Scale bar $=20 \mu \mathrm{m}$.

compared to PC-L therefore suggested a different PS-6-L uptake mechanism in RAW cells and consequently a different cellular distribution, which was investigated in more detail as described further on.

CLSM imaging of the near-infrared (NIR)-labeled lipids incorporated in the liposomal membrane revealed association of all types of liposomes with RAW cells (Figure 2e). No NIR autofluorescence signal was detected in RAW cells incubated without liposomes. In agreement with MRI, NIR fluorescence and therefore liposome

Table 3 Relaxivities of the liposomes in the cellular environment

\begin{tabular}{lcc}
\hline & Cellular $\mathbf{r}_{\mathbf{1}}\left(\mathrm{mM}^{\mathbf{- 1}} \cdot \mathbf{s}^{-\mathbf{1}}\right)$ & Cellular $\mathbf{r}_{\mathbf{2}}\left(\mathbf{m M}^{-\mathbf{1}} \cdot \mathbf{s}^{-\mathbf{1}}\right)$ \\
\hline PC-L & $1.9 \pm 0.3$ & $32.5 \pm 3.6$ \\
PS-6-L & $0.8 \pm 0.4$ & $16.3 \pm 5.8$ \\
PS-12-L & $1.4 \pm 0.5$ & $19.1 \pm 6.9$ \\
PS-37-L & $1.3 \pm 0.1$ & $22.0 \pm 8.1$ \\
\hline
\end{tabular}

association was highest for cells incubated with PS-6-L and intermediate for PC-L, while PS-12-L and PS-37-L showed similarly low levels of NIR fluorescence.

To confirm that the observed association of PS-6-L with RAW cells was mediated by their phagocytic character, endothelial H5V cells, for which no or minor phagocytosis was expected, were incubated with the different liposome formulations. MRI $\mathrm{T}_{1}$ and $\mathrm{T}_{2}$ maps for untreated and liposome-incubated cell pellets, shown in Figure 3a, revealed only minor differences between the various groups. Nevertheless, incubation with liposomes resulted in enhanced $R_{1}$ values ( $<<0.05$ vs. no $L$ ) and the highest values were detected for cells incubated with PS6 -L $\left(0.524 \pm 0.003 \mathrm{~s}^{-1}, \mathrm{p}<0.05\right.$ vs. all, Figure $\left.3 \mathrm{~b}\right)$. No significant differences were detected for $R_{2}$ ( $p>0.05$ vs. all). However, $R_{1}$ and $R_{2}$ values were significantly lower compared to RAW cell incubations for all groups ( $\mathrm{p}<0.05 \mathrm{vs.}$ RAW cells). $\Delta \mathrm{R}_{1}\left(=\mathrm{R}_{1, \mathrm{PS}-6-\mathrm{L}}-\mathrm{R}_{1, \text { no } \mathrm{L}}\right)$ values were 8.5 times lower for $\mathrm{H} 5 \mathrm{~V}$ cells compared to RAW cells, and for $\Delta \mathrm{R}_{2}$ this was even 45 times. CLSM of the H5V cells 

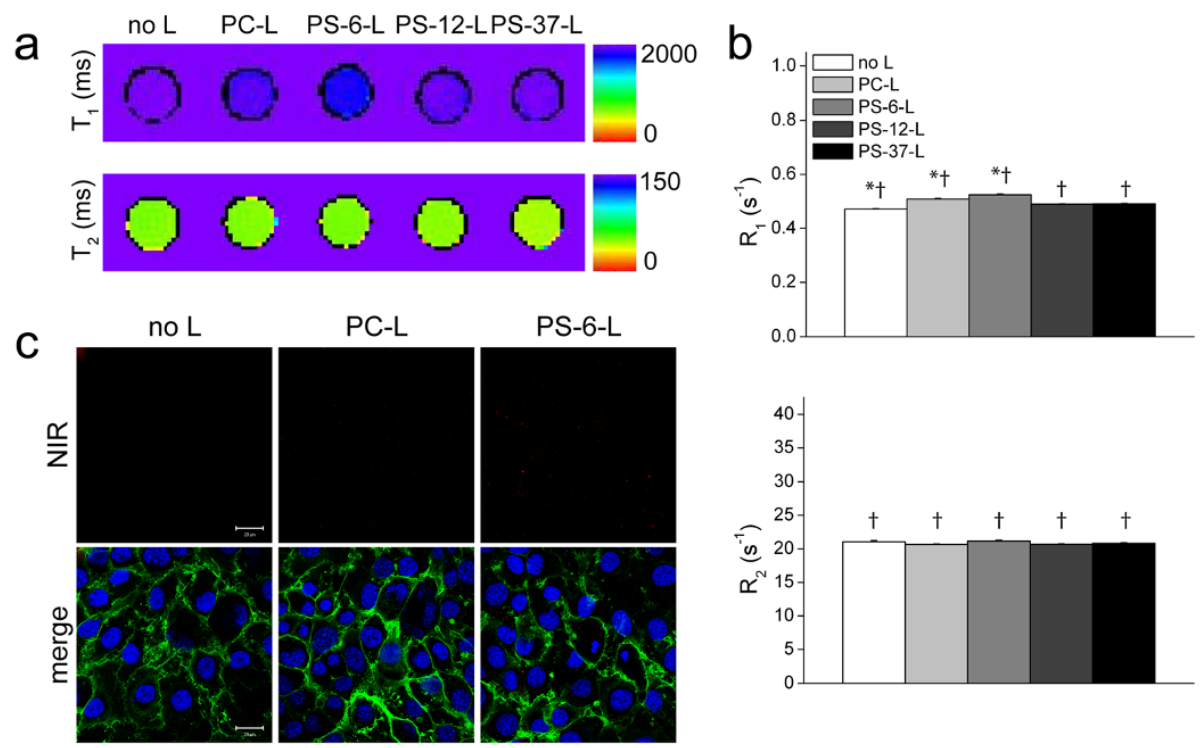

Figure 3 Association of PS-containing liposomes with H5V cells. a) Representative $T_{1}$ and $T_{2}$ maps of H5V cell pellets incubated with the different types of liposomes in RPMI medium. b) Average $R_{1}$ and $R_{2}$ of H5V cell pellets measured at $9.4 T$ ( $\left.n=3 / g r o u p\right)$. ${ }^{*}<<0.05$ vs. all, ANOVA with Bonferroni correction and $+p<0.05$ vs. RAW cell pellets, Student's t-test. c) CLSM images of H5V cells. Top row: in red, the fluorescence signal of the NIR-lipids present in liposomes. The laser intensity (20\% of maximal intensity) was ten times higher as compared to Figure $2 \mathrm{~d}$. Bottom row: NIR signal (in red) merged with signal of labeled H5V cell membranes (in green) and cell nuclei (in blue). Scale bar $=20 \mu \mathrm{m}$.

revealed no NIR fluorescence for incubations with PC-L and very few faint spots for PS-6-L (Figure 3c).

\section{Divalent cation dependency of liposome association with macrophages}

The above-described experiments suggested that liposomes with $6 \mathrm{~mol} \%$ DSPS (PS-6-L) were the most optimal formulation for targeting of macrophages. Therefore, PS-6-L was used in the experiments described from here. Association of PS-containing vesicles with the macrophage cell membrane depends on the presence of divalent cations such as $\mathrm{Ca}^{2+}$ and $\mathrm{Mg}^{2+}$ [11]. To test whether PS-6-L binding depended on the presence of divalent cations, which could be indicative for an interaction between these ions and PS resulting in membrane binding, RAW cells were incubated with PC-L and PS-6-L in Hank's buffered salt solution (HBSS) with $1.26 \mathrm{mM} \mathrm{Ca}^{2+}$ and $0.90 \mathrm{mM} \mathrm{Mg}^{2+}$ (HBSS+) or without $\mathrm{Ca}^{2+}$ and $\mathrm{Mg}^{2+}$ (HBSS-). Furthermore, samples from RAW cells incubated in incubation medium with intermediate $\mathrm{Ca}^{2+}$ and $\mathrm{Mg}^{2+}$ concentrations $(0.424 \mathrm{mM}$ and $0.407 \mathrm{mM}$, respectively), identical to the ones used in previous experiments, were included. Cellular association was quantified with FACS.

With increasing $\mathrm{Ca}^{2+}$ and $\mathrm{Mg}^{2+}$ concentrations, the FACS fluorescence intensities of RAW cells increased for both PC-L and PS-6-L (Figure 4a and 4b, respectively). The average NIR fluorescence for cells incubated with PC-L or PS-6-L in medium lacking $\mathrm{Ca}^{2+}$ and $\mathrm{Mg}^{2+}$ (HBSS-) was equal ( $p>0.05$, Figure $4 c$ ). Importantly, for incubations in medium with high $\mathrm{Ca}^{2+}$ and $\mathrm{Mg}^{2+}$ concentrations $(\mathrm{HBSS}+)$, fluorescence was significantly highest for PS-6-L ( $<<0.05$ vs. all).

DLS showed that the diameter of both PC-L and PS-6$\mathrm{L}$ increased after $2 \mathrm{~h}$ of incubation in HBSS+(Figure $4 \mathrm{~d}$ ). For PC-L the average hydrodynamic diameter changed from $164.3 \pm 0.9 \mathrm{~nm}$ in HBSS- to $209.5 \pm 25.2 \mathrm{~nm}$, while for PS-6-L the diameter increased from $104.6 \pm 19.4 \mathrm{~nm}$ to $170.8 \pm 33.5 \mathrm{~nm}$. This size increase could additionally enhance the uptake of both types of liposomes by the cells.

\section{Binding versus internalization}

To study whether PS-6-L were internalized by macrophages, RAW cells were incubated with PC-L or PS-6-L in HBSS+ at either $4^{\circ} \mathrm{C}$ or $37^{\circ} \mathrm{C}$. Incubation at $4^{\circ} \mathrm{C}$ inhibits phagocytosis and thus a comparison between $4^{\circ} \mathrm{C}$ or $37^{\circ} \mathrm{C}$ enabled a differentiation between binding to the cell membrane and internalization. FACS analysis of cells incubated at $4^{\circ} \mathrm{C}$ revealed no significant differences in average fluorescence intensities after incubation with PC-L and PS-6-L (Figure 5a-b). At $37^{\circ} \mathrm{C}$, however, a significantly higher fluorescence intensity was observed for PS-6-L (p $<0.05$ vs. all, Figure 5a-b).

CLSM confirmed the FACS measurements (Figure 5c). Incubation of RAW cells with PC-L and PS-6-L at $4^{\circ} \mathrm{C}$ resulted in minor association of liposomes. CLSM using higher laser intensities showed that the liposomes appeared as a rim around every cell, bound to the cell membrane. No significant internalization was observed. 

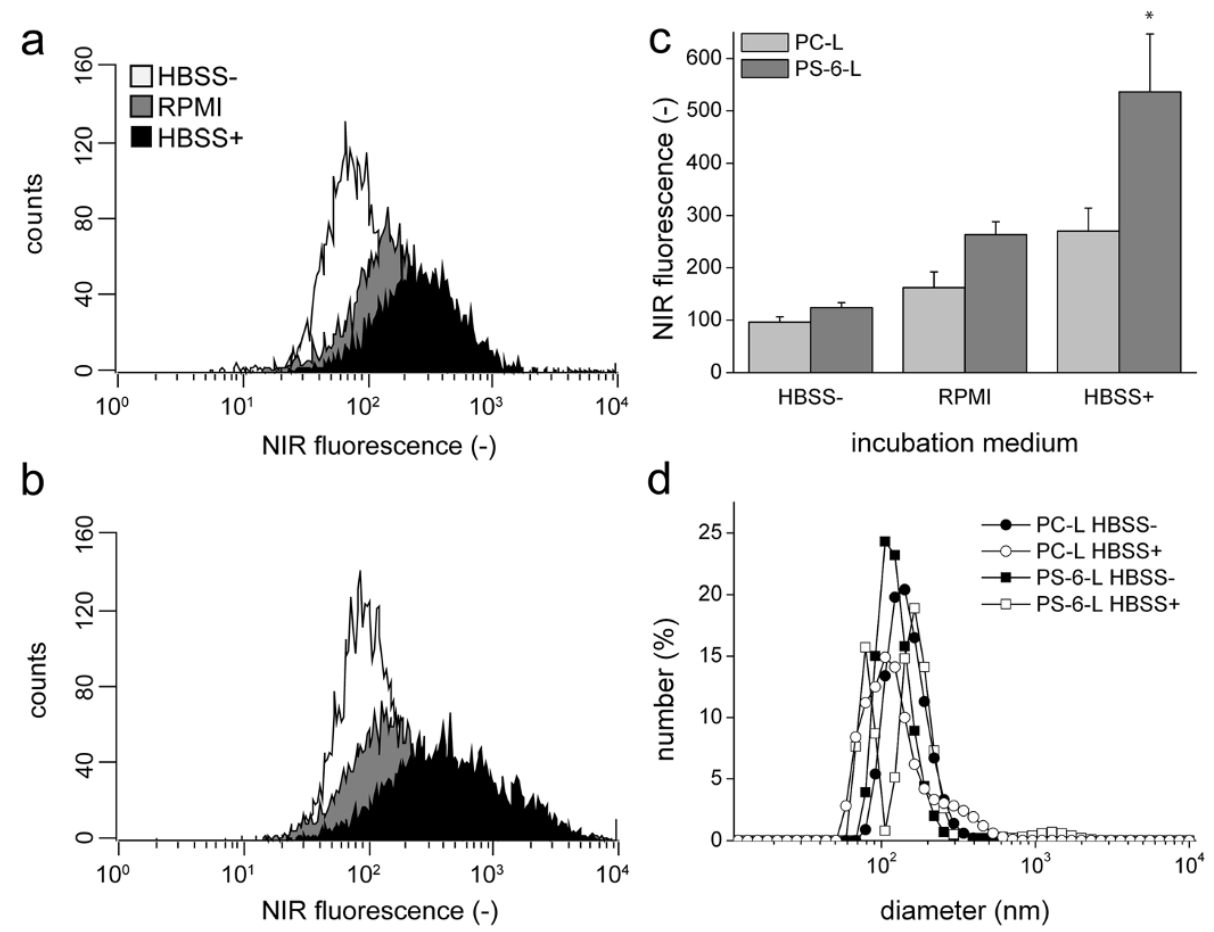

Figure 4 Divalent cation dependency of liposome association with RAW cells. Representative FACS spectra for RAW cells incubated with a) PC-L or b) PS-6-L in HBSS- without $\mathrm{Mg}^{2+}$ and $\mathrm{Ca}^{2+}$, RPMl incubation medium $\left(0.424 \mathrm{mM} \mathrm{Ca}^{2+}\right.$ and $0.407 \mathrm{mM} \mathrm{Mg}^{2+}$ ) or $\mathrm{HBSS}^{+}$with $1.26 \mathrm{mM} \mathrm{Mg}^{2+}$ and $0.90 \mathrm{mM} \mathrm{Ca}^{2+}$. c) Average fluorescence intensities for all samples ( $n=4 /$ group). ${ }^{*} p<0.05$ vs. all, ANOVA with Bonferroni correction. $\left.\mathbf{d}\right)$ Number-weighted DLS size distributions for PC-L and PS-6-L incubated in medium with and without $\mathrm{Ca}^{2+}$ and $_{\mathrm{Mg}^{2+}}\left(2 \mathrm{~h}, 37^{\circ} \mathrm{C}\right)$.

For incubations with $\mathrm{PC}-\mathrm{L}$ at $37^{\circ} \mathrm{C}$ CLSM images were comparable to incubations at $4^{\circ} \mathrm{C}$, with minor association of liposomes, and higher laser intensities revealed that PC-L were mainly bound to the cell membrane. CLSM confirmed that incubation with PS-6-L at $37^{\circ} \mathrm{C}$ resulted in massive internalization of the liposomes, as shown by the high NIR signal inside RAW cells.

\section{Discussion}

Macrophages play a decisive role in several cardiovascular diseases. For example, in atherosclerosis high macrophage content is one of the hallmarks of plaque vulnerability [2]. The inflammatory response after myocardial infarction is important for cardiac remodeling and outcome [1]. Therefore, macrophages form a significant therapeutic target in cardiovascular diseases and tools for noninvasive MR imaging of macrophages are highly desired. Iron oxides have been successfully applied for the MR visualization of macrophages in cardiovascular diseases [15-17]. Nevertheless, targeting of iron oxides to $\mathrm{CD} 11 \mathrm{~b} / \mathrm{CD} 18$, which is expressed on macrophages, did not improve specificity for MR imaging of macrophages in a mouse model of atherosclerosis [18]. Recently, Gd-labeled liposomes were used to visualize monocytes and/or macrophages infiltration in the mouse myocardium up to 7 days after myocardial infarction [19].
In this study, we describe the design and characterization of paramagnetic liposomes targeted to macrophages by incorporation of PS in the liposomal membrane. The liposomes contained Gd-DOTA-DSPE for MRI detection. Gd-DOTA-DSPE is a phospholipid that presents a high $r_{1}$ and the Gd-DOTA complex displays a high thermodynamic and kinetic stability [20]. As expected, at 9.4 T, the longitudinal relaxivity is not as high as at lower, clinical field strengths [20,21]. Importantly, incorporation of PS did not significantly affect liposomal $r_{1}$ and $r_{2}$ values. The $r_{2} / r_{1}$ ratio of the liposome formulations at $9.4 T$ was relatively high, which means that the liposomes will display a significant $\mathrm{T}_{2}$ effect as well. Nevertheless, by appropriately choosing the MRI sequence parameters, the $T_{1}$ effect of the liposomes can be effectively exploited (Figure 2).

A distinct difference between the PS-containing liposomes used in this study and previously reported formulations for use in in vivo MRI studies is the incorporation of $5 \mathrm{~mol} \%$ polyethylene glycol (PEG) lipids in the liposomal membrane. PEG reduces the interactions between the liposomes, reducing aggregation and ensuring a monodisperse formulation (Table 2 and Figure 1). Additionally, PEG increases the in vivo blood circulation half-life by reducing the interactions with plasma proteins, assuring a longer interaction time with 


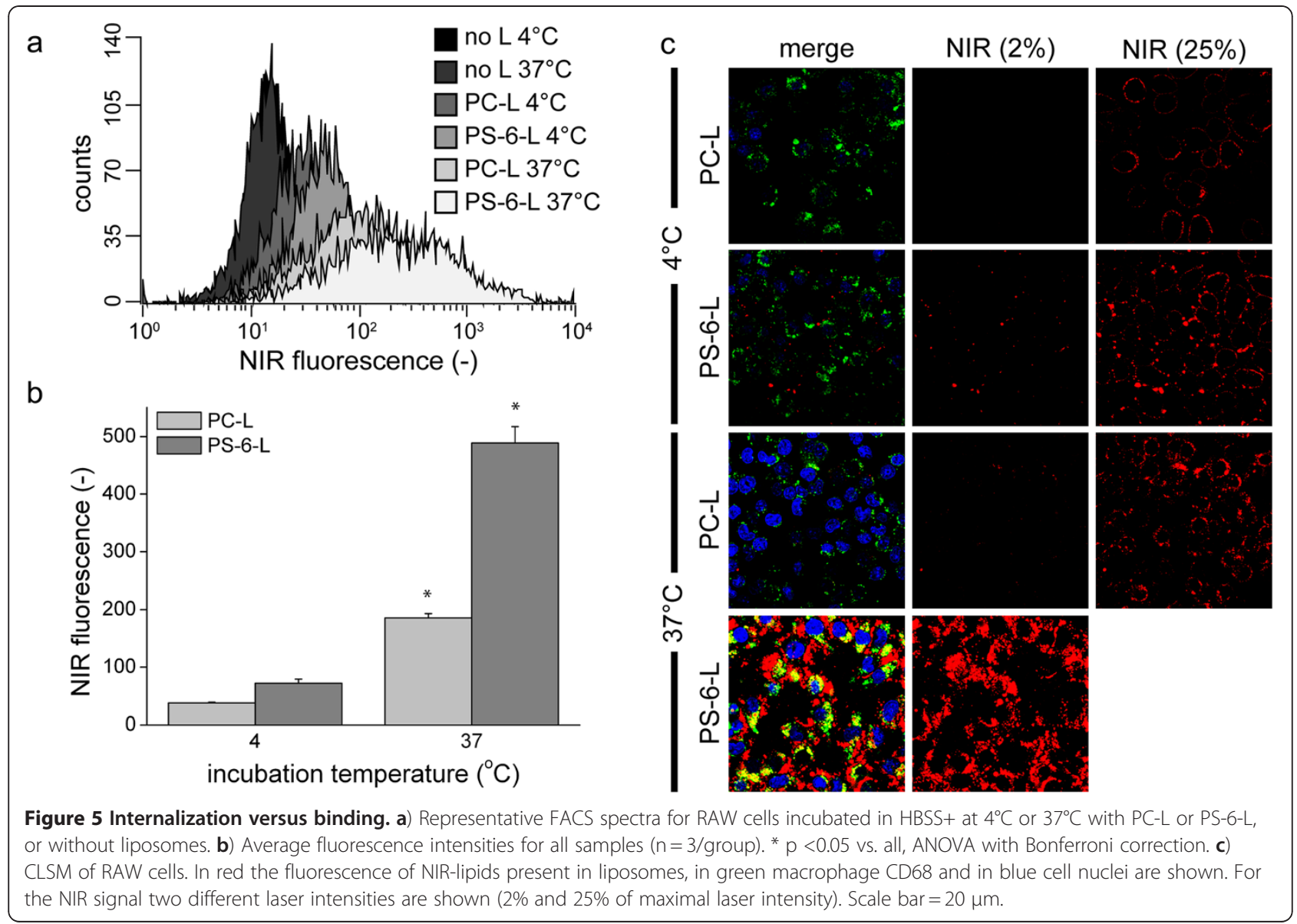

macrophages [5]. According to previous studies, incorporation of $5 \mathrm{~mol} \%$ PEG in PS-containing liposomes is not impeding the interaction of PS with macrophages, since at least $10-15$ mol\% PEG would be needed to completely shield the liposomes from any interactions with proteins [22-24]. We therefore did not expect a decrease in the uptake by shielding of the PS.

Liposomes containing 6 mol\% PS resulted in the highest uptake by RAW murine macrophages (Figure 2). Maiseyeu et al. and Rimle et al. have observed optimal uptake by macrophages of liposomes without PEG when these contained 5-12 mol\% PS [10,11]. Interestingly, these experimentally determined optimal concentrations are in the range of $2-10 \mathrm{~mol} \%$ PS found in the membranes of mammalian cells [25], which suggests that macrophages are optimally equipped to recognize and phagocytose nanoparticles that express approximate physiological concentrations of PS. Association was specific for macrophages as uptake by endothelial H5V cells was significantly lower (Figure 3).

Uptake of PS-containing liposomes by macrophages was stimulated by the presence of divalent cations (Figure 4). Higher uptake was not primarily caused by divalent cation-mediated clustering of the liposomes, since incubation of liposomes in $\mathrm{HBSS}+$ resulted in moderate changes in liposome size for both PC-L and PS-6-L. The HBSS+ buffer contained a physiologically relevant concentration of $1.26 \mathrm{mM} \mathrm{Ca}^{2+}$, compared to for example approximately $1.24 \mathrm{mM} \mathrm{Ca}^{2+}$ in mouse blood [26]. For the PS-mediated recognition of apoptotic cells by macrophages, different engulfment receptors have been identified, such as scavenger receptors, oxidized low-density lipoproteins recognizing receptors and CD68 [27], which for the LOX-1 scavenger receptor has been proven to be $\mathrm{Ca}^{2+}$-dependent [28]. Which of these receptors are important for PS-mediated uptake of liposomes remains unknown.

With respect to MR imaging of liposome uptake, a relatively high association of PS-6-L with macrophages, as determined with ICP-MS, resulted only in a modest increase in $R_{1}$ (Figure 2). This is probably related to compartmentalization of PS-6-L in intracellular vesicles after phagocytosis, which limits effective access of bulk water protons to the $\mathrm{Gd}$ contrast agent $[29,30]$. $\mathrm{T}_{1}$ shortening requires direct physical contact between $\mathrm{Gd}$ and water protons to be most effective. This interpretation is corroborated by the observation that the estimated cellular relaxivity of PS-6-L $\left(\mathrm{r}_{1}=0.8 \pm 0.4 \mathrm{mM}^{-1} \cdot \mathrm{s}^{-1}\right)$ was lower 
than the one of PS-6-L in aqueous solution $\left(\mathrm{r}_{1}=3.0 \pm 0.3 \mathrm{mM}^{-1} \cdot \mathrm{s}^{-1}\right)$. Furthermore, internalization of PS-6-L was observed by CLSM for incubations at $37^{\circ} \mathrm{C}$ (Figure 5).

The next step will be to apply and study the uptake of PS-6-L in a relevant animal model of cardiovascular inflammation, for example in atherosclerosis or myocardial infarction. Christiansen et al. have shown that echocardiography of PS-containing microbubbles trapped in infarcted myocardium correlated moderately well with MPO activity, which are excreted by inflammatory cells [31].

Apart from use in imaging applications, PS-containing liposomes are a promising vehicle for targeted drug delivery. Liposomes loaded with Q10, ATP or adenosine delivered to infarct myocardium were demonstrated to reduce infarct size and salvage ischemic myocardium [32-34]. Also, liposomes have been used as a vehicle for delivery of glucocorticoids drugs to perform anti-inflammatory cancer therapy [12]. Targeting could enhance the specificity of drug delivery to macrophages. Alternatively, PS-liposomes themselves can be used for therapy of inflammation as well [14,35-37]. As PS-liposomes mimic apoptotic cells, they inhibit pro-inflammatory cytokines release and promote secretion of anti-inflammatory cytokines. However, for therapy purposes higher PS-concentrations (up to $30 \mathrm{~mol} \%$ ) were used $[14,36]$, which in this study did not enhance uptake by macrophages.

\section{Conclusions}

In summary, paramagnetic liposomes, containing $6 \mathrm{~mol} \%$ of PS, showed enhanced uptake by macrophages compared to liposomes without PS, while significantly less uptake was observed for non-phagocytic cells. Association of PS-containing liposomes to macrophages was increased by the presence of divalent cations in the incubation medium and resulted mainly in internalization of liposomes, whereas only minor binding was observed. Therefore, these liposomes can be used for molecular MR imaging of macrophages and might as well be suitable for targeted drug delivery to macrophages in cardiovascular diseases.

\section{Methods}

\section{Preparation of PS-containing liposomes}

Liposomes containing different mole percentages of PS were prepared by modification of the protocol described by Hak et al. [20]. In short, lipid film hydration of a lipid mixture was performed (typically $50 \mu \mathrm{mol}$ of total lipid). The lipid mixture, consisting of 1,2-distearoyl-sn-glycero3-phospho-L-serine (DSPS, Avanti Polar Lipids, Alabaster, USA), 2-distearoyl-sn-glycero-3-phosphocholine (DSPC, Lipoid, Steinhausen, Switzerland), Gd-DOTA-1,2-distearoyl$s n$-glycero-3-phospoethanolamine (Gd-DOTA-DSPE, SyMO-
Chem BV, Eindhoven, the Netherlands), cholesterol (Avanti Polar Lipids) and 1,2-distearoyl-sn-glycero-3-phospoethanolamine- $N$-[methoxy(poly(ethylene glycol))-2000] (PEG2000-DSPE, Lipoid), was dissolved in chloroform and methanol $(8: 1 \mathrm{v} / \mathrm{v})$ at molar percentages as shown in Table 1. For liposomes containing DSPS, the mixture was heated to dissolve the DSPS (to maximally $65^{\circ} \mathrm{C}$ ). Additionally, $0.1 \mathrm{~mol} \%$ near-infrared664-1,2-distearoyl-sn-glycero-3-phospoethanolamine (NIR664-DSPE, SyMO-Chem $\mathrm{BV}$ ) was incorporated. After rotary evaporation at $30^{\circ} \mathrm{C}$ and overnight drying under a nitrogen flow, the lipid film was hydrated in HEPES-buffered saline (HBS, $10 \mathrm{mM}$ HEPES, $135 \mathrm{mM} \mathrm{NaCl}, \mathrm{pH} 7.4$ ) at $65^{\circ} \mathrm{C}$. The resulting multilamellar vesicles were sized by extrusion through $400 \mathrm{~nm}$ filters (2 times) and $200 \mathrm{~nm}$ filters (8 times). Finally, the liposomes were concentrated using ultracentrifugation (45 min, 55,000 rpm, $4^{\circ} \mathrm{C}$ ) and resuspended in HBS at a concentration of approximately $70 \mathrm{mM}$ total lipid.

\section{Characterization of liposomes}

Total lipid concentrations of the final liposome formulations were determined by a phosphate determination according to Rouser [38]. Hydrodynamic numberweighted size and size distribution were assessed with dynamic light scattering (DLS, ZetaSizer NanoS, Malvern Instruments, Worcestershire, UK) at $23^{\circ} \mathrm{C}$.

To confirm the presence of DSPS lipids in the PScontaining liposomes, thin layer chromatography (TLC) was performed on an aluminum sheet coated with silica gel $60 \mathrm{~F}_{254}$ (Merck BV, Schiphol-Rijk, the Netherlands) [39]. As eluent a mixture of chloroform, methanol, glacial acetic acid and water (65:25:8:4 v/v) was used. Liposomes were applied (expected concentrations of DSPS: PC-L $0 \mathrm{mg} / \mathrm{mL}$, PS-6-L $2 \mathrm{mg} / \mathrm{mL}$, PS- $12-\mathrm{L} 4 \mathrm{mg} / \mathrm{mL}$ and PS$37-\mathrm{L} 6 \mathrm{mg} / \mathrm{mL}$ ) and allowed to migrate for $30 \mathrm{~min}$. As controls standard solutions of DSPS $(0.5,1,2,4$ and $8 \mathrm{mg} / \mathrm{mL}$ ) were used. Finally, primary and secondary amines in DSPS and Gd-DOTA-DSPE were detected with ninhydrin.

Liposomal morphology was evaluated with cryogenic transmission electron microscopy (cryoTEM). Samples were vitrified on carbon-coated cryoTEM grids with a vitrification robot (Vitrobot Mark III, FEI, Hillsboro, USA). Imaging was performed on a Tecnai 20 Sphera TEM instrument (FEI) equipped with a LaB6 filament $(200 \mathrm{kV})$ and Gatan cryoholder (approximately $-170^{\circ} \mathrm{C}$ ) at 25,000x magnification.

Liposomal longitudinal and transversal relaxation times $\left(\mathrm{T}_{1}\right.$ and $\mathrm{T}_{2}$ ) were determined with a 9.4 $\mathrm{T}$ small animal MR scanner (Bruker Biospin GmbH, Ettlingen, Germany) equipped with a 35-mm-diameter quadrature birdcage RF coil (Rapid Biomedical, Rimpar, Germany). For $\mathrm{T}_{1}$ measurements an inversion recovery fast low angle shot (FLASH) sequence was used, with the following 
parameters: overall repetition time (TR) $15 \mathrm{sec}$, TR $4 \mathrm{~ms}$, echo time (TE) $2 \mathrm{~ms}$, flip angle $(\alpha) 15^{\circ}$, number of excitations (NEX) 4, field of view (FOV) $3 \times 3 \mathrm{~cm}^{2}$, matrix $128 \times 128,1 \mathrm{~mm}$ slice thickness, 32 segments and 60 inversion times ranging from 72 to $4792 \mathrm{~ms}$. $\mathrm{T}_{2}$ relaxation times were determined using a multi-slice multi-echo sequence with the following parameters: TR $2000 \mathrm{~ms}, 32$ TEs ranging from 9 to $288 \mathrm{~ms}, \alpha 180^{\circ}$, NEX 4, FOV $3 \times 3$ $\mathrm{cm}^{2}$, matrix $128 \times 128$ and $1 \mathrm{~mm}$ slice thickness. $\mathrm{T}_{1}$ and $\mathrm{T}_{2}$ relaxation times were calculated by mono-exponential fitting with a custom-built fitting program (Mathematica 6, Wolfram Research Europe, Oxfordshire, UK). Relaxivities $r_{1}$ and $r_{2}$ (in $\mathrm{mM}^{-1} \cdot \mathrm{s}^{-1}$ ) were determined from $R_{i}=$ $R_{i, 0}+r_{i} \cdot[G d]$, with $i=1,2, R_{i}=1 / T_{i}, R_{i, 0}$ the relaxation rate of a sample without liposomes and [Gd] between 0.001 and $1 \mathrm{mM} \mathrm{Gd}$.

\section{Cell culture}

Mouse macrophages, RAW264.7 (European Collection of Animal Cell Cultures (ECACC)), were cultured in RPMI medium (phenol-red free), supplemented with 10\% FBS, $2 \mathrm{mM}$ L-glutamine and $100 \mathrm{U} / \mathrm{mL}$ penicillin/ streptomycin. As non-phagocytic control cells, murine heart endothelioma cells, H5V (kindly provided by prof. dr. G. Molema, University of Groningen, the Netherlands), were maintained in DMEM medium, containing $10 \%$ FBS, $2 \mathrm{mM} \mathrm{L-glutamine} \mathrm{and} 100 \mathrm{U} / \mathrm{mL}$ penicillin/ streptomycin [40].

\section{Association of PS-containing liposomes with RAW cells}

To determine the mol\% of PS present in liposomes resulting in maximal uptake by macrophages, RAW cells were incubated with PC-L, PS-6-L, PS-12-L and PS-37-L for $2 \mathrm{~h}$ at $37^{\circ} \mathrm{C}(1 \mathrm{mM}$ total lipid). For MRI and inductively coupled plasma mass spectrometry (ICP-MS), cells were harvested by scraping and non-bound liposomes were removed by centrifugation ( $3 \times 5 \mathrm{~min}, 500 \mathrm{~g}$, RPMI medium at $\left.37^{\circ} \mathrm{C}\right)$. Cells were fixed in $4 \%$ PFA $(250 \mu \mathrm{L})$ and a loosely packed cell pellet was allowed to form by storage at $4^{\circ} \mathrm{C}$ ( $>2$ days). For confocal laser scanning microscopy (CLSM), cells were cultured on coverslips. After incubation with the liposome formulations, cells were fixed with 4\% PFA (20 min). Finally cells were washed with and stored in phosphate bufferd saline (PBS).

\section{Association of PS-containing liposomes with H5V cells}

To confirm that PS-containing liposomes were not taken up by endothelial cells, H5V cells were incubated with PC-L, PS-6-L, PS-12-L and PS-37-L for $2 \mathrm{~h}$ at $37^{\circ} \mathrm{C}$ (1 $\mathrm{mM}$ total lipid). For MRI, cells were washed with medium $\left(37^{\circ} \mathrm{C}\right)$ and $\mathrm{PBS}\left(37^{\circ} \mathrm{C}\right)$. Afterwards, cells were harvested with trypsin/EDTA, fixed with $4 \%$ PFA and a loosely packed pellet was allowed to form. For CLSM, cells were cultured on gelatin-coated coverslips and handled as described above.

\section{Divalent cation dependency of liposome association with RAW cells}

The association of liposomes to RAW cells under different calcium and magnesium concentrations was studied. RAW cells were incubated with PC-L and PS-6-L (2 h, $37^{\circ} \mathrm{C}, 1 \mathrm{mM}$ total lipid) in Hank's buffered salt solution (HBBS) containing $1.26 \mathrm{mM} \mathrm{Ca}^{2+}$ and $0.90 \mathrm{mM} \mathrm{Mg}^{2+}$ (HBSS+), HBSS without $\mathrm{Ca}^{2+}$ and $\mathrm{Mg}^{2+}$ (HBSS-) and RPMI medium (0.424 $\mathrm{mM} \mathrm{Ca}^{2+}$ and $0.407 \mathrm{mM} \mathrm{Mg}^{2+}$ ). Afterwards, cells were harvested by scraping, washed in the appropriate medium (HBSS+, HBSS- or RPMI, $37^{\circ} \mathrm{C}$ ), fixed in $4 \%$ PFA $(20 \mathrm{~min})$ and stored in $0.01 \%$ sodiumazide in PBS for FACS.

To investigate possible clustering of PC-L and PS-6-L under high calcium and magnesium concentrations, liposomes were incubated in HBSS + or HBSS- $\left(2 \mathrm{~h}, 37^{\circ} \mathrm{C}\right)$. Changes in hydrodynamic number-weighted diameter and size distribution were measured with DLS as described above at $37^{\circ} \mathrm{C}$.

\section{Binding versus internalization}

To evaluate phagocytosis of PS-containing liposomes by macrophages, RAW cells were incubated with PC-L or PS-6-L at $4^{\circ} \mathrm{C}$ or at $37^{\circ} \mathrm{C}(1 \mathrm{mM}$ total lipid in HBSS+, $2 \mathrm{~h})$. Incubation at $4^{\circ} \mathrm{C}$ inhibits phagocytosis. For FACS, cells were incubated with liposomes in HBSS+ and harvested and washed as described above. For CLSM, cells were cultured and incubated with liposomes in microscopy chambers (Ibidi GmbH, München, Germany). Afterwards, cells were washed with $\mathrm{HBSS}+\left(4^{\circ} \mathrm{C}\right.$ or $\left.37^{\circ} \mathrm{C}\right)$, fixed with 4\% PFA (20 min), washed and stored in PBS.

\section{Cellular relaxation rates and relaxivities}

The cellular relaxation rates of cell pellets $\left(R_{1}\right.$ and $\left.R_{2}\right)$ were determined at 9.4 $\mathrm{T}$ using the MRI protocol as described above. Furthermore, the cell pellet volume was determined using a 3D FLASH sequence with the following parameters: TR $25 \mathrm{~ms}$, TE $3.7 \mathrm{~ms}, \alpha 30^{\circ}$, NEX 1 , FOV 25.6x25.6x25.6 $\mathrm{mm}^{3}$ and matrix 256x256x256. Cell pellets were segmented with OsiriX Imaging Software (www.osirix-viewer.com) and pellet volumes were calculated. The Gd content of cell pellets was determined with ICP-MS (DRCII, Perkin Elmer, Waltham, USA) after destruction in nitric acid and perchloric acid $(1: 2 \mathrm{v} / \mathrm{v})$ at $180^{\circ} \mathrm{C}$. Next, gadolinium concentrations were derived using the cell pellet volume. Cellular relaxivities were calculated from $R_{i}=R_{i, 0}+r_{i} \cdot[G d]$, with $i=1,2$, and $R_{i, 0}$ the relaxation rate of untreated cells. 


\section{Cellular fluorescence quantification}

The fluorescence intensity of NIR664-lipids, present in the liposomes associated with RAW cells, was quantified by fluorescence activated cell sorting (FACS) on a Guava Easycyte 8HT (Millipore, Billerica, USA). NIR664 was excited with a $640 \mathrm{~nm}$ laser and detected using a 661/19 $\mathrm{nm}$ band-pass filter. Mean cellular fluorescence intensity was calculated with GuavaSoft 1.0 software (Millipore) and was corrected for autofluorescence as detected by measurement of untreated cells.

\section{Cellular CLSM}

The cellular distribution of liposomes was studied with CLSM. Cell membranes of RAW cells were labeled with rat anti-mouse CD68-fluorescein isothiocynate (CD68FITC, $4 \mu \mathrm{g} / \mathrm{mL}$, AbD Serotec, Dusseldorf, Germany). H5V cells were labeled with rat anti-mouse CD31 $(10 \mu \mathrm{g} / \mathrm{mL}$, BioLegend, Uithoorn, The Netherlands) conjugated to goat anti-rat FITC (Invitrogen, Bleiswijk, The Netherlands). Nuclei were stained with 4/6-diamidino-2-phenylindole dihydrochloride (DAPI, $0.1 \mu \mathrm{g} / \mathrm{mL}$, Invitrogen).

A Zeiss LSM META system (Carl Zeiss BV, Sliedrecht, the Netherlands) was used for acquisition of CLSM images. NIR664 present in liposomes was excited with a $633 \mathrm{~nm}$ HeNe laser $(5.0 \mathrm{~mW})$ and the emission was filtered with a 680/60 nm band-pass filter. Cell membranes labeled with FITC were excited with a $488 \mathrm{~nm}$ Ar laser and the emission was filtered with a 525/50 nm bandpass filter. For two-photon excitation of DAPI, a Ti:Sapphire laser tuned to $780 \mathrm{~nm}$ was used and emission was captured with a 460/50 band-pass filter. All images were acquired with a $63 \mathrm{x} / 1.4$ oil immersion objective, a matrix of $2048 \times 2048$, resulting in a resolution of $0.07 \times 0.07 \mu \mathrm{m}^{2}$, and 4 averages.

\section{Statistics}

All data are presented as mean \pm standard error of the mean (SEM). To test for significant differences between groups, one-way analysis of variance (ANOVA) with Bonferroni correction for multiple group comparisons or a Student's t-test for independent samples was applied. All statistical analyses were performed in PASW Statistics 18.02 (IBM Corporation, Armonk, NY, USA) and P $<0.05$ was considered significant.

\footnotetext{
Abbreviations

ANOVA: Analysis of variance; CLSM: Confocal laser scanning microscopy; cryoTEM: Cryogenic transmission electron microscopy; DAPI: 4/6-diamino-2phenylindole dihydrochloride; DSPC: 1,2-distearoyl-sn-glycero-3phospocholine; DSPS: 1,2-distearoyl-sn-glycero-3-phospho-L-serine; DSPE: 1,2distearoyl-sn-glycero-3-phospoethanolamine; DLS: Dynamic light scattering; EPR: Enhanced permeability and retention; FACS: Fluorescence activated cell sorting; FBS: Fetal bovine serum; FITC: Fluorescein isothiocynate; Gd: Gadolinium; HBS: HEPES buffered saline; HBSS: Hank's buffered salt solution; HBSS-: HBSS without $\mathrm{Ca}^{2+}$ and $\mathrm{Mg}^{2+} ; \mathrm{HBSS}+$ : HBSS with $\mathrm{Ca}^{2+}$ and $\mathrm{Mg}^{2+}$; ICP-MS: Inductively coupled plasma mass spectrometry; L: Liposomes; MRI: Magnetic resonance imaging; NIR: Near-infrared; PBS: Phosphate
}

buffered saline; PC: Phosphatidylcholine; PDI: Polydispersity index; PEG: Polyethylene glycol; PFA: Paraformaldehyde; PS: Phosphatidylserine; PS$x$-L: Liposomes containing $x$ mol\% of PS; $r_{1}$ : Longitudinal relaxivity; $r_{2}$ : Transversal relaxivity; $R_{1}$ : Longitudinal relaxation rate; $R_{2}$ : Transversal relaxation rate; $S E M$ : Standard error of the mean; $T_{1}$ : Longitudinal relaxation time; $\mathrm{T}_{2}$ : Transversal relaxation time; TLC: Thin layer chromatography.

\section{Competing interests}

The authors declare that they have no competing interests.

\section{Authors' contributions}

All authors added intellectual content, read and approved the final manuscript. TG: Designed the study, performed experiments, performed data analysis, performed statistical analysis, prepared and edited the manuscript. SY, LP, LS: Performed experiments, performed data analysis, edited the manuscript. KN: Co-designed the study, edited the manuscript. GS: Principal investigator, designed the study, prepared and edited the manuscript.

\section{Acknowledgments}

This work is supported by the Dutch Technology Foundation STW, applied science division of NWO and the Technology Program of the Ministry of Economic Affairs; Grant number: 07952. We thank Henk Keijzer (SyMO-Chem BV, Eindhoven, the Netherlands) for assistance with the TLC measurements.

Received: 21 June 2012 Accepted: 23 August 2012

Published: 28 August 2012

\section{References}

1. Frangogiannis NG: The immune system and cardiac repair. Pharmacol Res 2008, 58:88-111.

2. Glass CK, Witztum JL: Atherosclerosis: the road ahead. Cell 2001, 104:503-516.

3. Abraham SA, Waterhouse DN, Mayer LD, Cullis PR, Madden TD, Bally MB: The liposomal formulation of doxorubicin. Methods Enzymol 2005, 391:71-97.

4. Gabizon A, Shmeeda H, Barenholz Y: Pharmacokinetics of pegylated liposomal doxorubicin: review of animal and human studies. Clin Pharmacokinet 2003, 42:419-436.

5. Allen TM: Long-circulating (sterically stabilized) liposomes for targeted drug delivery. Trends Pharmacol Sci 1994, 15:215-220.

6. van Bochove GS, Paulis LEM, Segers D, Mulder WJM, Krams R, Nicolay K, Strijkers GJ: Contrast enhancement by differently sized paramagnetic MRI contrast agents in mice with two phenotypes of atherosclerotic plaque. Contrast Media Mol Imaging 2011, 6:35-45.

7. Geelen T, Paulis LE, Coolen BF, Nicolay K, Strijkers GJ: Passive targeting of paramagnetic lipid-based contrast agents to acute mouse cardiac ischemia/reperfusion injury. In Proceedings of the 19th Annual Meeting ISMRM; Montreal, Canada, 2011:1356.

8. Hengartner MO: The biochemistry of apoptosis. Nature 2000, 407:770-776.

9. Fadok VA, Voelker DR, Campbell PA, Cohen JJ, Bratton DL, Henson PM: Exposure of phosphatidylserine on the surface of apoptotic lymphocytes triggers specific recognition and removal by macrophages. J Immunol 1992, 148:2207-2216.

10. Maiseyeu A, Mihai G, Kampfrath T, Simonetti OP, Sen CK, Roy S, Rajagopalan S, Parthasarathy S: Gadolinium containing phosphatidylserine liposomes for molecular imaging of atherosclerosis. J Lipid Res 2009, 50:2157-2163.

11. Rimle D, Derski W, Petty HR: Enhanced binding of phosphatidylserinecontaining lipid vesicle targets to RAW264 macrophages. Mol Cell Biochem 1984, 64:81-87.

12. Strijkers GJ, Kluza E, van Tilborg GAF, van der Schaft DWJ, Griffioen AW, Mulder WJM, Nicolay K: Paramagnetic and fluorescent liposomes for target-specific imaging and therapy of tumor angiogenesis. Angiogenesis 2010, 13:161-173.

13. Kluza E, Yeo SY, Schmid S, van der Schaft DWJ, Boekhoven RW, Schiffelers RM, Storm G, Strijkers GJ, Nicolay K: Anti-tumor activity of liposomal glucocorticoids: the relevance of liposome-mediated drug delivery, intratumoral localization and systemic activity. J Control Release 2011, 151:10-17.

14. Harel-Adar T, Ben Mordechai T, Amsalem Y, Feinberg MS, Leor J, Cohen S: Modulation of cardiac macrophages by phosphatidylserine-presenting 
liposomes improves infarct repair. Proc Natl Acad Sci U S A 2011, 108:1827-1832.

15. Yang Y, Yang Y, Yanasak N, Schumacher A, Hu TC-C: Temporal and noninvasive monitoring of inflammatory-cell infiltration to myocardial infarction sites using micrometer-sized iron oxide particles. Magn Reson Med 2010, 63:33-40.

16. Sosnovik DE, Nahrendorf M, Deliolanis N, Novikov M, Aikawa E, Josephson L, Rosenzweig A, Weissleder R, Ntziachristos V: Fluorescence tomography and magnetic resonance imaging of myocardial macrophage infiltration in infarcted myocardium in vivo. Circulation 2007, 115:1384-1391.

17. Jaffer FA, Libby P, Weissleder R: Molecular Imaging of cardiovascular disease. Circulation 2007, 116:1052-1061.

18. von zur Muhlen C, Fink-Petri A, Salaklang J, Paul D, Neudorfer I, Berti V Merkle A, Peter K, Bode C, von Elverfeldt D: Imaging monocytes with iron oxide nanoparticles targeted towards the monocyte integrin MAC-1 (CD11b/CD18) does not result in improved atherosclerotic plaque detection by in vivo MRI. Contrast Media Mol Imaging 2010, 5:268-275.

19. Naresh NK, Xu Y, Klibanov AL, Vandsburger MH, Meyer CH, Leor J, Kramer $\mathrm{CM}$, French BA, Epstein FH: Monocyte and/or macrophage infiltration of heart after myocardial infarction: MR imaging by using $\mathrm{T}_{1}$-shortening liposomes. Radiology 2012, 264:428-435.

20. Hak S, Sanders HMHF, Agrawal P, Langereis S, Grüll H, Keizer HM, Arena F, Terreno E, Strijkers GJ, Nicolay K: A high relaxivity Gd(III)DOTA-DSPE-based liposomal contrast agent for magnetic resonance imaging. Eur J Pharm Biopharm 2009, 72:397-404.

21. Strijkers GJ, Mulder WJM, Van Heeswijk RB, Frederik PM, Bomans P, Magusin PCMM, Nicolay K: Relaxivity of liposomal paramagnetic MRI contrast agents. MAGMA 2005, 18:186-192.

22. Chiu G, Bally M, Mayer L: Selective protein interactions with phosphatidylserine containing liposomes alter the steric stabilization properties of poly(ethylene glycol). Biochim Biophys Acta 2001, 1510:56-59.

23. Johnstone SA, Masin D, Mayer L, Bally MB: Surface-associated serum proteins inhibit the uptake of phosphatidylserine and poly(ethylene glycol) liposomes by mouse macrophages. Biochim Biophys Acta 2001, 1513:25-37.

24. Levchenko TS, Rammohan R, Lukyanov AN, Whiteman KR, Torchilin VP: Liposome clearance in mice: the effect of a seperate and combined presence of surface charfe and polymer coating. Int J Pharmaceut 2002, 240:95-102.

25. Vance JE, Steenbergen R: Metabolism and functions of phosphatidylserine. Prog Lipid Res 2005, 44:207-234.

26. Tordoff M, Bachmanov A, Reed D: Forty mouse strain survey of voluntary calcium intake, blood calcium, and bone mineral content. Physiol Behav 2007, 91:632-643.

27. Ravichandran KS: Beginnings of a good apoptotic meal: the find-me and eat-me signalling pathways. Immunity 2011, 35:445-455.

28. Murphy JE, Tacon D, Tedburry PR, Hadden JM, Knowling S, Sawamura T, Peckham M, Philips SE, Walker JH, Ponnambalam S: LOX-1 scavenger receptor mediates calcium-dependent recognition of phosphatidylserine and apoptotic cells. Biochem J 2006, 393:107-115.

29. Strijkers GJ, Hak S, Kok MB, Springer CS, Nicolay K: Three-compartment $T_{1}$ relaxation model for intracellular paramagnetic contrast agents. Magn Reson Med 2009, 61:1049-1058.

30. Kok MB, Hak S, Mulder WJM, van der Schaft DWJ, Strijkers GJ, Nicolay K: Cellular compartmentalization of internalized paramagnetic liposomes strongly influences both $\mathrm{T}_{1}$ and $\mathrm{T}_{2}$ Relaxivity. Magn Reson Med 2009, 61:1022-1032.

31. Christiansen JP, Leong-Poi H, Klibanov AL, Kaul S, Lindner JR: Noninvasive imaging of myocardial reperfusion injury using leukocyte-targeted contrast echocardiography. Circulation 2002, 105:1764-1767.

32. Verma DD, Hartner WC, Levchenko TS, Bernstein EA, Torchilin VP: ATP-loaded liposomes effectively protect the myocardium in rabbits with an acute experimental myocardial infarction. Pharm Res 2005 , 22:2115-2120

33. Verma DD, Hartner WC, Thakkar V, Levchenko TS, Torchilin VP: Protective effect of coenzyme Q10-loaded liposomes on the myocardium in rabbits with an acute experimental myocardial infarction. Pharm Res 2007, 24:2131-2137.

34. Takahama H, Minamino $T$, Asanuma H, Fujita M, Asai T, Wakeno M, Sasaki H, Kikuchi $\mathrm{H}$, Hashimoto K, Oku N, et al: Prolonged targeting of ischemic/ reperfused myocardium by liposomal adenosine augments cardioprotection in rats. J Am Coll Cardiol 2009, 53:709-717.

35. Huynh MN, Fadok VA, Henson PM: Phosphatidylserine-dependent ingestion of apoptotic cells promotes TGF- $\beta 1$ secretion and the resolution of inflammation. J Clin Invest 2002, 109:41-50.

36. Hoffmann PR, Kench JA, Vondracek A, Kruk E, Daleke DL, Jordan M, Marrack $P$, Henson PM, Fadok VA: Interaction between phosphatidylserine and the phosphatidylserine receptor inhibits immune responses in vivo. $J$ Immunol 2005, 174:1393-1404.

37. Ramos GC, Fernandes D, Charão $C T$, Souza DG, Teixeira MM, Assreuy J: Apoptotic mimicry: phosphatidylserine liposomes reduce inflammation through activation of peroxisome proliferator-activated receptors (PPARs) in vivo. Br J Pharmacol 2007, 151:844-850.

38. Rouser G, Fleischer S, Yamamoto A: Two dimensional thin layer chromatographic separation of polar lipids and determination of phospholipids by phosphorus analysis of spots. Lipids 1970, 5:494-496

39. Skipski VP, Peterson RF, Barclay M: Separation of phosphatidyl ethanolamine, phosphatidyl serine, and other phospholipids by thinlayer chromatography. J Lipid Res 1962, 3:467-470.

40. Garlanda C, Parravicini C, Sironi M, De Rossi M, de Calmanovici Wainstock R, Carozzi F, Bussolino F, Colotta F, Mantovani A, Vecchi A: Progressive growth in immunodeficient mice and host cell recruitment by mouse endothelial cells transformed by polyoma middle-sized T antigen: implications for the pathogenesis of opportunistic vascular tumors. ProC Natl Acad Sci U S A 1994, 91:7291-7295.

doi:10.1186/1477-3155-10-37

Cite this article as: Geelen et al:: Internalization of paramagnetic phosphatidylserine-containing liposomes by macrophages. Journal of Nanobiotechnology 2012 10:37.

\section{Submit your next manuscript to BioMed Central and take full advantage of:}

- Convenient online submission

- Thorough peer review

- No space constraints or color figure charges

- Immediate publication on acceptance

- Inclusion in PubMed, CAS, Scopus and Google Scholar

- Research which is freely available for redistribution
C Biomed Central 